\title{
Monitoring of temporal trend of atmospheric metals by moss Hypnum cupressiforme Hedw in Jammu (India)
}

\author{
Dinesh K. Saxena, Sachin Agarwal ${ }^{b}$, A Saxena ${ }^{c}$, Seema Kodesia ${ }^{a}$ \\ ${ }^{a}$ Department of Botany, Bareilly College, Bareilly, India \\ c. University of Waterloo, Canada \\ ${ }^{b}$ Research Scholar Environmental Sciences, IGNOU, New Delhi
}

\begin{abstract}
Atmospheric pollutants, such as metals, are extremely variable in space and time and it is cumbersome and expensive to deduce detailed information over a vast area using traditional instruments. Consequently, a cost-effective bio-monitor was preferred in the present study to analyze the atmospheric metal load. For this purpose, a moss Hypnum cupressiforme Hedw was inducted amongst moss species available to evaluate the intensity and trend of atmospheric deposition of $\mathrm{Zn}, \mathrm{Cu}, \mathrm{Cd}$, and $\mathrm{Pb}$ in Jammu in the state of Jammu and Kashmir, India for a period of three years, 20142016. The biomonitoring experimentation was performed by transplanting moss bags prepared from Hypnum cupressiforme, after validating its tolerance against metals using a photosynthetic efficiency analyzer in the field. Moss Hypnum cupressiforme Hedw exposed seasonally, covering summer, monsoon, and winter periods, upon the analysis provided time-integrated patterns of metal bioavailability at the study sites. An attempt was made to compare the seasonal variations during the three years of study and findings exhibited significant seasonal variations in metal. The gradient of the metal load was in the order of $\mathrm{Cu}>\mathrm{Cd}>\mathrm{Zn}>\mathrm{Pb}$. The total average percentage increase in the metals under consideration, $\mathrm{Zn}, \mathrm{Pb}, \mathrm{Cu}$, and $\mathrm{Cd}$, was $40 \%, 37 \%, 79 \%$, and $76 \%$, respectively during three subsequent years. It is worth mentioning here that biomonitoring studies on atmospheric metals have never been explored for this region.
\end{abstract}

Keywords: Biomonitoring, atmospheric metals, moss bag transplants, temporal trend, Hypnum cupressiforme

\section{Introduction}

Air pollution is one of the most significant problems in the world caused by the rapid increase in population, energy consumption, and industrial activities that may result in major threats to human health and the environment.

The study was undertaken in Jammu city (Fig. 1), which is the principal city in the Jammu division and also, the winter capital of Jammu and Kashmir. It is located at $32.73^{\circ} \mathrm{N}, 74.87^{\circ} \mathrm{E}$ on the banks of the Tawi river which is delimited mostly in the Himalayan mountains and shares its border with Himachal Pradesh and Punjab. The city of Jammu, located in the north of India, was chosen for the present study because there is an inflow of 6.4 million pilgrims and tourists in the city every year besides its population of 1.6 million, as of 2016 (Fig. 1). Due to the increased number of pilgrims, there is heavy congestion of the automobiles on road, resulting in a many-fold increase in atmospheric pollutants due to emissions spewed out from various sources, including these automobiles.

${ }^{*}$ Corresponding author. Tel.: + 09457874240

Fax: +9876543210; E-mail: Author.dinesh.botany@gmail.com

(C) 2016 International Association for Sharing Knowledge and Sustainability

DOI: $10.5383 /$ ijtee.17.02.006
Although several plant species such as lichens $[1,2]$ mosses [3,4], ferns [5], grasses, and trees [6] are used for biomonitoring. Mosses have a major advantage over other groups of plants. Lichen is not suited for monitoring, as it is difficult to take it out or remove it from substratum or rock. On the other hand, as algae grow in water, algal analysis cannot reflect the pollution load of air. Angiosperms, gymnosperms and ferns, all have roots buried inside the soil, hence, their analysis may reflect the soil metal load $[9,10]$.

Widespread occurrence, capability to survive under extreme environmental conditions, and availability throughout the year make mosses an ideal plant for monitoring. In addition, they lack roots and water-conducting tissues, consequently, their analysis does not reflect the soil metal load [7]. The specific biology including the thin epidermis makes them capable to receive and accumulate chemical substances, predominantly from the surrounding atmosphere including industrial sites without any selectivity parameter [8,9]. Moss provides integration of deposition over the exposure period, thus, 
making them useful, simple, and inexpensive monitors [10]. Bryo-monitoring has the added advantage of being relatively simple and inexpensive and can be used as both passive [11, 13] and active acceptors [12] in addition to retrospective monitoring i.e., past atmospheric metal data [14]. Trace elements may be absorbed in the moss from the atmosphere either as soluble chemical species in wet deposition or contained in particles from dry deposition.

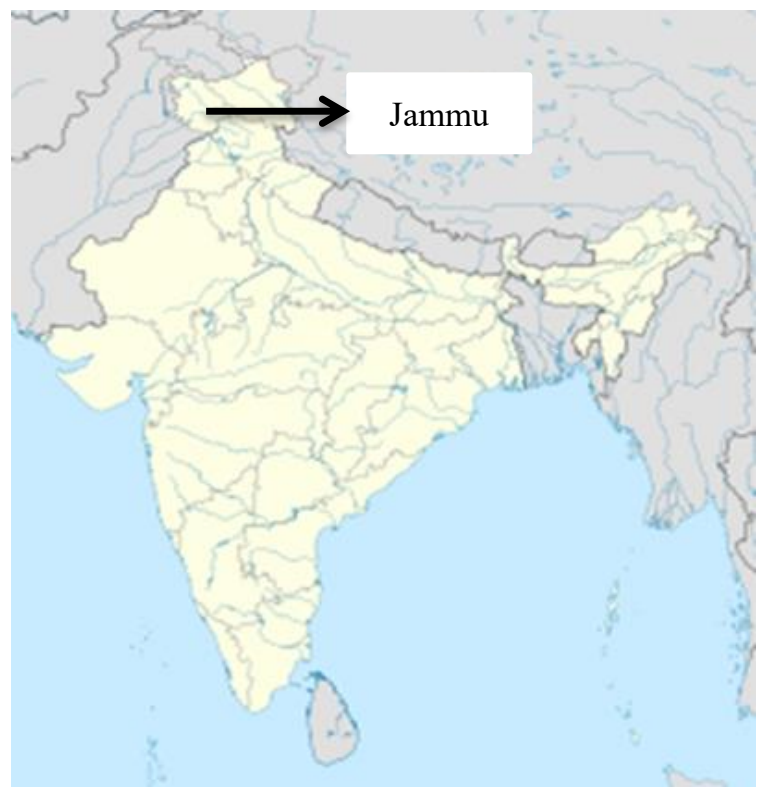

Fig. 1 Map of Jammu (India)

Mosses have also several advantages over conventional techniques of atmospheric deposition of metals $[10,15]$. The high surface to mass ratio of mosses is effective in trapping airborne particles using both native and transplant methods [16]. Metal deposits in moss at very high concentrations because they pick up nutrients directly from ambient air and retains them for quite a long time without impairing its physiological process due to metal-binding protein [17]. Therefore, their analysis is a reflection of atmospheric metal load. Bryo-monitoring is simple, relatively inexpensive, employing both, spatial and temporal trends of metal accumulation $[18,20]$ and allows exploring a greater number of sampling sites including relative to absolute [19] with considerable flexibility in choosing sampling sites and excludes the effect of different lifetime metal history of test plants, which is an important limitation in passive biomonitoring. A study on the evaluation of some commonly available biological monitors for heavy metal pollution by using moss bags was discussed by Sloof et al [21] and Ares et al [22].

Amongst commonly available moss species, the best metal tolerant moss species, Hypnum cupressiforme was inducted for biomonitoring after validating for metal tolerance using a noninvasive approach by measuring chlorophyll fluorescence parameters. Chlorophyll fluorescence measurement is useful to quantify abiotic stress response, accordingly, confirming the stress tolerance species quickly in the field [23]. It is also known as Integrated Biomarker Response (IBR) because of the simple, practical, and accommodating approach for various stress applications [24] or measurable indicators of some health status. The same can be measured at different locations of surveys, at different periods, or for various types of samples, depending on the comparisons needed. This approach has never been opted earlier. The objectives of the present survey are:

1. To characterize quantitatively and qualitatively the atmospheric deposition of metals

2. To assess the gradient of metal deposition concerning important metal emission sources and to identify the polluted areas

3. To produce counter maps of regional deposition patterns

4. Seasonal and annual trend of metal load for 2014 to 2016.

\section{Methodology}

A biomonitoring campaign with moss-bags was accomplished in Jammu city to study if the geography affects urban air pollution.

\subsection{Collection of moss specimens}

After critical evaluation of some commonly used biological monitors for heavy metal pollution [52], available five moss species were collected from the field and were identified based on taxonomical examination with the help of information available in the literature [25, 26, 27, 28, 41]. Our findings revealed that amongst available moss species one of the species (Hypnum cupressiforme) was found in most urban sites (common in distribution) and confirms its acceptability to grow in the areas of study. Therefore, it can serve as a biomonitor of atmospheric metal pollution within these areas. Consequently, widely distributed mosses, Hypnum cupressiforme Hedw was collected from a pristine background of Manesar and inducted in a monitoring program after validation of tolerance against metals. Nonetheless, moss Hylocomium species, a favorite for monitoring, were excluded because they were less widely distributed.

\subsection{Screening for tolerant moss species in the lab by measuring Chl. fluorescence signals:}

Thudium sp, Hypnum sp, Polytrichum sp, Tortulla sp, and Isopterigium $\mathrm{sp}$ moss species were collected from the field and tested for their health status (Table 1) as well as for tolerance against metals like $\mathrm{Cu}, \mathrm{Cd}, \mathrm{Pb}$, and $\mathrm{Zn}$ (Tables 2(a)-2(d)). The non-invasive approach was applied to validate tolerant moss species by measuring chlorophyll fluorescence signals [29]. If the plant is not well or diseased or in stress, its physiology will be affected, consequently, it is not able to convert all energy in $\mathrm{C}$ fixation, therefore, there is loss of energy in the form of chlorophyll fluorescence [30]. Change in Chl. fluorescence emission is an indication of impaired health [31]. The first step in the measurement process is to cover the sample area of the moss, with small lightweight leaf clips provided by the manufacturer. The clip has a small shutter plate that should be closed over the leaf when the clip is fixed over the leaf, meaning light is excluded, and dark adaptation takes place. After an interruption of the light period of 30 minutes, the clips shutter was opened to measure after pressing the sensor button. All values (Fo, Fm, Fv) were recorded by PEA. The Fv/Fm ratio has, thus, been considered as a sensitive indicator of plant photosynthetic efficiency during stress [30].

Table 1. Moss species measured for chlorophyll fluorescence $(F v / F m)$ at different sites (rural and urban) of Jammu 


\begin{tabular}{|l|c|c|c|c|}
\hline \multicolumn{1}{|c|}{ Moss species } & Forest cover & Forest roadside & Urban site 2 km & Bus stand \\
\hline Hypnum sp & 0.763 & 0.760 & 0.687 & 0.653 \\
\hline Thuidium & 0.751 & 0.747 & 0.505 & 0.446 \\
\hline Drepanocladus & 0.689 & 0.669 & 0.514 & 0.442 \\
\hline Polytricum & 0.651 & 0.603 & 0.422 & 0.372 \\
\hline Dicranum & 0.649 & 0.536 & 0.393 & 0.347 \\
\hline Bryum & 0.563 & 0.545 & 0.347 & 0.322 \\
\hline Hylocomium & 0.775 & 0.704 & 0.478 & 0.307 \\
\hline Brachythecium & 0.69 & 0.658 & 0.475 & 0.409 \\
\hline Cratoneuron & 0.672 & 0.621 & 0.403 & 0.299 \\
\hline Isoptergium spb & 0.726 & 0.601 & 0.551 & 0.583 \\
\hline
\end{tabular}

Values are represented as mean $\pm S E$.

The significance test of ANOVA and DMRT has been done at a $1 \%$ and $5 \%$ significance level.

Values superscripted with the same alphabets in a horizontal row are not significantly different at $1 \%$ and $5 \%$ significance levels at different sites.

*Values in a horizontal row are significantly different at a 5\% significance level.

Table 2a. Photosynthetic efficiency (Fv/Fm) measured in mosses in the field of Manesar, Jammu at different concentrations of $\mathrm{Zn}$

\begin{tabular}{|l|l|l|l|l|l|}
\hline $\begin{array}{l}\text { S. } \\
\text { No. }\end{array}$ & Moss & Control & $\begin{array}{l}10 \\
\text { ppm } \\
\text { conc. }\end{array}$ & $\begin{array}{l}20 \\
\text { ppm } \\
\text { conc. }\end{array}$ & $\begin{array}{l}40 \\
\text { ppm } \\
\text { conc. }\end{array}$ \\
\hline 1 & Hypnum sp & 0.789 & 0.769 & 0.731 & 0.607 \\
\hline 2 & Thuidium sp & 0.793 & 0.754 & 0.708 & 0.512 \\
\hline 3 & Brachythecium sp & 0.721 & 0.682 & 0.623 & 0.489 \\
\hline 4 & Hylocomium sp & 0.705 & 0.670 & 0.619 & 0.456 \\
\hline 5 & Isoptergium sp & 0.730 & 0.659 & 0.607 & 0.432 \\
\hline
\end{tabular}

Table 2b. Photosynthetic efficiency (Fv/Fm) measured in mosses in the field of Manesar, Jammu at different concentrations of $\mathrm{Pb}$

\begin{tabular}{|c|l|c|c|c|c|}
\hline $\begin{array}{c}\text { S. } \\
\text { No. }\end{array}$ & Moss & Control & $\begin{array}{l}10 \\
\mathrm{ppm} \\
\text { conc. }\end{array}$ & $\begin{array}{l}\text { 20ppm } \\
\text { conc. }\end{array}$ & $\begin{array}{l}40 \\
\mathrm{ppm} \\
\text { conc. }\end{array}$ \\
\hline 1 & Hypnum $\mathrm{sp}$ & .777 & .716 & .653 & .518 \\
\hline 2 & Thuidium $\mathrm{sp}$ & .763 & .718 & .668 & .512 \\
\hline 3 & $\begin{array}{l}\text { Brachythecium } \\
\text { sp }\end{array}$ & .706 & .702 & .607 & .472 \\
\hline 4 & Hylocomium $\mathrm{sp}$ & .705 & .604 & .606 & .462 \\
\hline 5 & Isoptergium $\mathrm{sp}$ & .700 & .693 & .592 & .457 \\
\hline
\end{tabular}

Table 2c. Photosynthetic efficiency ( $\mathrm{Fv} / \mathrm{Fm})$ measured in mosses in the field of Manesar, Jammu at different concentrations of $\mathrm{Cu}$.

\begin{tabular}{|l|l|c|c|c|c|}
\hline $\begin{array}{l}\text { S. } \\
\text { No }\end{array}$ & Moss & $\begin{array}{l}\text { Oppm } \\
\text { conc. }\end{array}$ & $\begin{array}{l}10 \mathrm{pp} \\
\mathrm{m} \\
\text { conc. }\end{array}$ & $\begin{array}{l}\text { 20ppm } \\
\text { conc. }\end{array}$ & $\begin{array}{l}40 \\
\mathrm{ppm} \\
\text { conc. }\end{array}$ \\
\hline 1 & Hypnum $\mathrm{sp}$ & .761 & .739 & .672 & .603 \\
\hline 2 & Thuidium $\mathrm{sp}$ & .753 & .732 & .686 & .626 \\
\hline 3 & $\begin{array}{l}\text { Brachythecium } \\
\text { sp }\end{array}$ & .696 & .676 & .604 & .576 \\
\hline 4 & $\begin{array}{l}\text { Hylocomium } \\
\text { sp }\end{array}$ & .663 & .616 & .556 & .489 \\
\hline 5 & Isoptergium $\mathrm{sp}$ & .653 & .634 & .569 & .517 \\
\hline
\end{tabular}

Table 2d. Photosynthetic efficiency (Fv/Fm) measured in mosses in the field of Manesar, Jammu at different concentrations of $\mathrm{Cd}$

\begin{tabular}{|l|l|l|l|l|l|}
\hline S. No. & Moss & Control & $\begin{array}{l}10 \mathrm{ppm} \\
\text { conc. }\end{array}$ & $\begin{array}{l}20 \mathrm{ppm} \\
\text { conc. }\end{array}$ & $\begin{array}{l}40 \mathrm{ppm} \\
\text { conc. }\end{array}$ \\
\hline 1 & Hypnum sp & 0.727 & 0.721 & 0.660 & 0.653 \\
\hline 2 & Thuidium sp & 0.733 & 0.723 & 0.598 & 0.542 \\
\hline 3 & $\begin{array}{l}\text { Brachythecium } \\
\mathrm{sp}\end{array}$ & 0.696 & 0.743 & 0.552 & 0.518 \\
\hline 4 & $\begin{array}{l}\text { Hylocomium } \\
\mathrm{sp}\end{array}$ & 0.685 & 0.679 & 0.545 & 0.513 \\
\hline 5 & Isoptergium $\mathrm{sp}$ & 0.630 & 0.689 & 0.515 & 0.486 \\
\hline
\end{tabular}

\subsection{Moss bag transplants}

Validated tolerant moss Hypnum cupressiforme Hedw was collected along the Manesar lake (Forest cover) treated as control or background values of Jammu and was brought to the laboratory in plastic bags. Mosses were washed and were surface dried.

Nylon moss bags of $10 \times 8$ inches size [15] were prepared and filled with freshly collected $6 \mathrm{~g}$ moss Hypnum cupressiforme Hedw. Moss transplants were made in triplicate, cross sectionwise in all four directions (east, west, north, and south) at equidistance of $0.5 \mathrm{~km}, 1.0 \mathrm{~km}, 3.0 \mathrm{Km}$ from Gandhi Nagar Govt. hospital at nearly the same height of $15 \mathrm{ft}$. After the exposure period of 4 months (representing one season), moss transplants were harvested for metal analysis and in its place fresh moss bags were exposed for another four months covering another season, Similar process was followed over the 3 years of the study period (2014-2016).

\subsection{Cleaning, drying digestion and analysis of moss samples}

Transplant moss samples were sorted carefully and other plants or plant parts such as seeds, needles, and grasses were removed $[15,32]$. The sorted moss samples were washed with tap water many times to remove unwanted material and final washing was done with distilled water at least 2-3 times. Brown parts were taken out and only green parts were dried to constant mass at $30-35^{\circ} \mathrm{C}$. The dried plant material $(1 \mathrm{~g})$ was digested in $5 \mathrm{~mL}$ of analytical grade concentrated $\mathrm{HNO}_{3}$ and $2 \mathrm{~mL} \mathrm{HClO}_{4}$. 
The digested samples were filtered using Whatman No. 42 filter paper. Samples were analyzed by atomic absorption spectrophotometer at Electronic Corporation of India Ltd (ECIL) using the protocol (chem.science.unideb.hu/Pharm/FAAS.pdf). A blank was run to check for possible contamination during extraction. Metals undertook (zinc, copper, cadmium, lead) were analyzed by using respective hollow cathode lamps (imported) purchased from ECIL, Hyderabad.

\subsection{Preparations of herbarium records}

Voucher herbarium envelopes were prepared. Dried moss specimens (4-6 in number only) from each transplant bag were kept on filter paper and placed in voucher envelopes for the record. These voucher specimens are used in the future to compare the metal data of present with past specimens as done during the pandemic period [32]. Each voucher herbarium envelope was assigned a temporary herbarium number, family, genera, species, place of collection, and GPS data. Same was deposited in the "Bryophyte Environmental Bank" which was established in Botany Department, Bareilly College, Bareilly in 2001 [33]. ANOVA test $(\mathrm{p}<0.01,0.05)$ was performed to compare the metal concentration at different seasons and distances by utilizing Dunkun's Multiple Range Test [34]. Summary statistics were used to obtain the mean and standard error [35]. Cartographic representation of the results was performed with the program package Surfer (Golden Software Inc., U. S. A.).

\section{Results}

Moss Hypnum cupressiforme Hedw was collected from a tree trunk along the lake site and applied as a biomonitor of atmospheric metal deposition and to differentiate various sources and estimate the regional differences. Moss was robust, light green to brown, glossy, and procumbent. Branches were pinnate. Paraphyllia were very few $[26,36]$. Leaves were imbricate, concave ovate, or oblong-lanceolate. Margins were smooth or faintly denticulate at the tip. Costa was short. Leaf cells were linear, alar cells are well developed by a large number of quadrate to sub-quadrate cells $[36,43]$

Seasonally exposed moss bags of Hypnum cupressiforme were harvested after 4 every month and were analyzed for 4 metals, given in Table $3 \mathrm{a}-3 \mathrm{~d}$, and the concentrations of the heavy metals were compared with those obtained from the previous surveys in 2014 and 2015. Moss bag of Hypnum cupressiforme harvested from catchments sites of Jammu during summer, exhibited maximum $\mathrm{Zn}$ values in the proximity of west $0.5 \mathrm{~km}$ during the year 2014 and 2016, whereas, the same was measured in its minimum concentration in the north at the distance of $3 \mathrm{~km}$. Moss bags belonging to the monsoon season harvested at the distance of 0.5 and $3 \mathrm{~km}$, respectively, in 2014 , 2015, and 2016, exhibited consistently highest and lowest values of zinc towards west and east of Jammu (Table 3a). Seasonally harvested moss bags of all study sites exhibited seasonally significant difference $(\mathrm{p} \leq 0.01,0.05)$ in $\mathrm{Zn}$ concentration as compared with metal analyzed from transplants of baseline i.e., the control site. Results of $\mathrm{Pb}$ contamination analyzed from moss bags show significantly different values over baseline concentration nearly at all the sites during all the three consecutive years (Table $3 b$ ). Significant differences $(p<0.01)$ were calculated at a few transplant sites between summer and winter and during monsoon and winter seasons. The highest annual average distribution pattern of lead was $1.061 \mathrm{mg} \mathrm{g}^{1}{ }^{1} \mathrm{DW}$ (2014), 1.212 mg g- ${ }^{1}$ DW (2015), and $1.493 \mathrm{mg} \mathrm{g-1} \mathrm{DW} \mathrm{(2016)} \mathrm{at} \mathrm{the} 0.5 \mathrm{~km}$ moss sampling distance towards the west. Lead values declined up to $0.474 \mathrm{mg} \mathrm{g}-1 \mathrm{DW}, 0.571 \mathrm{mg} \mathrm{g}-1 \mathrm{DW}$, and $0.693 \mathrm{mg} \mathrm{g}-1$ DW during 2014, 2005, and 2016 respectively, in proximity to $3 \mathrm{~km}$ towards the east (Fig. $2 \mathrm{a}-2 \mathrm{c}$ ).

Table 3a. Seasonal variations in zinc $\left(\mathrm{mg} \mathrm{g}^{-1} \mathrm{DW}\right)$ in moss Hypnum cupressiforme Hedw from different transplant sites of Jammu during 2014, 2015 \& 2016

\begin{tabular}{|c|c|c|c|c|c|c|c|c|c|}
\hline \multirow{2}{*}{$\begin{array}{l}\text { Sampling } \\
\text { Sites }\end{array}$} & \multicolumn{3}{|c|}{ Summer } & \multicolumn{3}{|c|}{ Monsoon } & \multicolumn{3}{|c|}{ Winter } \\
\hline & 2014 & 2015 & 2016 & 2014 & 2015 & 2016 & 2014 & 2015 & 2016 \\
\hline $\begin{array}{l}\text { Baseline } \\
\text { (Manesar) }\end{array}$ & $0.146 \pm 0.011$ & $0.204 \pm 0.058$ & $0.299 \pm 0.057$ & $0.018 \pm 0.005^{\mathrm{a}}$ & $0.027 \pm 0.005^{\mathrm{b}}$ & $0.051 \pm 0.006^{\mathrm{c}}$ & $0.034 \pm 0.015^{\mathrm{a}}$ & $0.043 \pm 0.012^{\mathrm{b}}$ & $0.073 \pm 0.012^{\mathrm{c}}$ \\
\hline $\begin{array}{l}\text { North } \\
(0.5 \mathrm{~km})\end{array}$ & $0.828 \pm 0.023$ & $0.905 \pm 0.058^{\mathrm{a}}$ & $1.059 \pm 0.044^{\mathrm{b}}$ & $0.515 \pm 0.028^{c}$ & $0.508 \pm 0.015$ & $0.614 \pm 0.028$ & $0.635 \pm 0.044^{c}$ & $0.722 \pm 0.053^{\mathrm{a}}$ & $0.987 \pm 0.102^{b}$ \\
\hline $\begin{array}{l}\text { North } \\
(1.0 \mathrm{~km})\end{array}$ & $0.881 \pm 0.044$ & $* 1.051 \pm 0.051$ & $1.137 \pm 0.056$ & $0.506 \pm 0.028$ & $0.547 \pm 0.056$ & $* 0.708 \pm 0.044$ & $0.694 \pm 0.032$ & $* 0.764 \pm 0.074$ & $* 0.931 \pm 0.088$ \\
\hline $\begin{array}{l}\text { North } \\
(3.0 \mathrm{~km})\end{array}$ & $\begin{array}{c}* 0.762 \pm 0.03 \\
5\end{array}$ & $0.836 \pm 0.048^{\mathrm{a}}$ & $\begin{array}{c}0.958 \pm 0.045^{b} \\
\mathrm{c}\end{array}$ & $0.534 \pm 0.019$ & $0.615 \pm 0.048$ & $\begin{array}{c}0.754 \pm 0.072^{b} \\
d\end{array}$ & $\begin{array}{c}* 0.605 \pm 0.05 \\
7\end{array}$ & $0.657 \pm 0.086^{\mathrm{a}}$ & $0.807 \pm 0.029^{d}$ \\
\hline $\begin{array}{l}\text { South } \\
(0.5 \mathrm{~km})\end{array}$ & $0.784 \pm 0.017$ & $1.011 \pm 0.071$ & $1.182 \pm 0.086^{\mathrm{a}}$ & $0.464 \pm 0.045^{b}$ & $0.654 \pm 0.058^{c}$ & $* 0.832 \pm 0.040$ & $\begin{array}{c}0.604 \pm 0.042 \\
\text { b }\end{array}$ & $0.806 \pm 0.056^{c}$ & $\begin{array}{c}* 1.038 \pm 0.026 \\
\text { a }\end{array}$ \\
\hline $\begin{array}{l}\text { South } \\
(1.0 \mathrm{~km})\end{array}$ & $\begin{array}{c}* 1.250 \pm 0.07 \\
8\end{array}$ & $1.706 \pm 0.100$ & $2.182 \pm 0.087$ & $0.756 \pm 0.032$ & $0.934 \pm 0.071$ & $1.109 \pm 0.027$ & $\begin{array}{c}* 1.113 \pm 0.04 \\
7\end{array}$ & $1.308 \pm 0.057$ & $1.456 \pm 0.028$ \\
\hline $\begin{array}{l}\text { South } \\
(3.0 \mathrm{~km})\end{array}$ & $0.956 \pm 0.042$ & $* 1.187 \pm 0.089$ & $* 1.372 \pm 0.036$ & $0.641 \pm 0.028^{a}$ & $0.807 \pm 0.016$ & $0.991 \pm 0.100$ & $0.731 \pm 0.029^{\mathrm{a}}$ & $* 0.913 \pm 0.054$ & $* 1.132 \pm 0.058$ \\
\hline $\begin{array}{ll}\text { East } & (0.5 \\
\mathrm{km}) & \end{array}$ & $1.054 \pm 0.086^{\mathrm{a}}$ & $* 1.268 \pm 0.082$ & $1.610 \pm 0.061$ & $0.335 \pm 0.043$ & $0.663 \pm 0.057$ & $0.861 \pm 0.072$ & $0.918 \pm 0.024^{\mathrm{a}}$ & $* 1.044 \pm 0.048$ & $1.312 \pm 0.061$ \\
\hline $\begin{array}{ll}\text { East } & (1.0 \\
\mathrm{km}) & \end{array}$ & $1.217 \pm 0.024$ & $1.496 \pm 0.113$ & $1.778 \pm 0.069$ & $\begin{array}{c}* 0.737 \pm 0.01 \\
4\end{array}$ & $* 0.891 \pm 0.046$ & $1.030 \pm 0.019$ & $\begin{array}{c}* 0.893 \pm 0.05 \\
8\end{array}$ & $* 1.111 \pm 0.063$ & $1.457 \pm 0.101$ \\
\hline $\begin{array}{ll}\text { East } & (3.0 \\
\mathrm{km}) & \end{array}$ & $\begin{array}{c}* 0.792 \pm 0.04 \\
5\end{array}$ & $* 0.964 \pm 0.056$ & $1.148 \pm 0.068$ & $0.317 \pm 0.026$ & $0.462 \pm 0.032^{\mathrm{a}}$ & $* 0.563 \pm 0.031$ & $\begin{array}{c}* 0.594 \pm 0.02 \\
9\end{array}$ & $\begin{array}{c}* 0.692 \pm 0.015 \\
\text { a }\end{array}$ & $* 0.841 \pm 0.043$ \\
\hline $\begin{array}{l}\text { West }(0.5 \\
\mathrm{km})\end{array}$ & $1.380 \pm 0.036$ & $1.735 \pm 0.118$ & - & $\begin{array}{c}* 0.914 \pm 0.02 \\
7\end{array}$ & $1.077 \pm 0.057^{\mathrm{a}}$ & $1.256 \pm 0.028$ & $\begin{array}{c}* 1.058 \pm 0.01 \\
0\end{array}$ & $1.237 \pm 0.026^{\mathrm{a}}$ & $1.558 \pm 0.012$ \\
\hline $\begin{array}{l}\text { West }(1.0 \\
\mathrm{km})\end{array}$ & $1.183 \pm 0.077$ & $1.415 \pm 0.089$ & $1.742 \pm 0.095$ & $0.717 \pm 0.059$ & $0.807 \pm 0.031^{\mathrm{a}}$ & $0.907 \pm 0.053$ & $0.917 \pm 0.030$ & $1.018 \pm 0.057^{\mathrm{a}}$ & $1.212 \pm 0.104$ \\
\hline $\begin{array}{l}\text { West }(3.0 \\
\mathrm{km})\end{array}$ & $0.781 \pm 0.047$ & $\begin{array}{c}* 0.907 \pm 0.054 \\
\text { a }\end{array}$ & $1.016 \pm 0.028^{b}$ & $0.516 \pm 0.024^{c}$ & $\begin{array}{c}* 0.613 \pm 0.063 \\
\mathrm{~d}\end{array}$ & $0.693 \pm 0.030^{\mathrm{e}}$ & $0.586 \pm 0.033^{c}$ & $0.737 \pm 0.073^{\mathrm{ad}}$ & $0.864 \pm 0.059^{\text {be }}$ \\
\hline
\end{tabular}


Table 3b. Seasonal variations in Lead ( $\left.\mathrm{mg} \mathrm{g}^{-1} \mathrm{DW}\right)$ in moss Hypnum cupressiforme from different transplant sites in Jammu during 2014, 2015 \& 2016

\begin{tabular}{|c|c|c|c|c|c|c|c|c|c|}
\hline \multirow{2}{*}{$\begin{array}{l}\text { Sampling } \\
\text { Sites }\end{array}$} & \multicolumn{3}{|c|}{ Summer } & \multicolumn{3}{|c|}{ Monsoon } & \multicolumn{3}{|c|}{ Winter } \\
\hline & 2014 & 2015 & 2016 & 2014 & 2015 & 2016 & 2014 & 2015 & 2016 \\
\hline Baseline & $0.102 \pm 0.057$ & $0.173 \pm 0.009$ & $0.202 \pm 0.058$ & $0.010 \pm 0.002^{\mathrm{a}}$ & $0.018 \pm 0.005^{\mathrm{b}}$ & $0.035 \pm 0.012^{\mathrm{c}}$ & $0.023 \pm 0.001^{\mathrm{a}}$ & $0.033 \pm 0.012^{\mathrm{b}}$ & $0.051 \pm 0.011^{\mathrm{c}}$ \\
\hline $\begin{array}{l}\text { North }(0.5 \\
\mathrm{km})\end{array}$ & $0.747 \pm 0.021$ & $0.833 \pm 0.032$ & $0.956 \pm 0.101$ & $* 0.374 \pm 0.035$ & $0.459 \pm 0.045$ & $* 0.509 \pm 0.045$ & $* 0.506 \pm 0.057$ & $0.615 \pm 0.047$ & $* 0.712 \pm 0.059$ \\
\hline $\begin{array}{l}\text { North }(1.0 \\
\mathrm{km})\end{array}$ & $0.811 \pm 0.024$ & $0.917 \pm 0.059$ & $* 1.039 \pm 0.013$ & $0.459 \pm 0.020$ & $0.519 \pm 0.068$ & $0.562 \pm 0.029$ & $0.581 \pm 0.045$ & $0.691 \pm 0.028$ & $* 0.762 \pm 0.069$ \\
\hline $\begin{array}{l}\text { North }(3.0 \\
\mathrm{km})\end{array}$ & $0.754 \pm 0.057$ & $0.809 \pm 0.042$ & $0.883 \pm 0.060^{\mathrm{a}}$ & $0.505 \pm 0.028^{\mathrm{b}}$ & $0.563 \pm 0.041^{\mathrm{c}}$ & $0.734 \pm 0.054^{\mathrm{ad}}$ & $0.556 \pm 0.015^{\mathrm{b}}$ & $0.581 \pm 0.025^{\mathrm{c}}$ & $0.643 \pm 0.059^{\text {bd }}$ \\
\hline $\begin{array}{l}\text { South }(0.5 \\
\mathrm{km})\end{array}$ & $0.713 \pm 0.014$ & $0.773 \pm 0.035^{\mathrm{a}}$ & $0.887 \pm 0.060^{\mathrm{b}}$ & $0.437 \pm 0.030^{c}$ & $0.510 \pm 0.015^{\mathrm{d}}$ & $* 0.585 \pm 0.029$ & $0.558 \pm 0.031^{\mathrm{c}}$ & $0.633 \pm 0.041^{\mathrm{ad}}$ & $* 0.785 \pm 0.042^{b}$ \\
\hline $\begin{array}{l}\text { South }(1.0 \\
\mathrm{km})\end{array}$ & $* 1.242 \pm 0.103$ & $1.434 \pm 0.070$ & $1.806 \pm 0.057$ & $0.687 \pm 0.029$ & $0.779 \pm 0.013$ & $0.959 \pm 0.072$ & $* 1.008 \pm 0.075$ & $1.180 \pm 0.086$ & $1.271 \pm 0.094$ \\
\hline $\begin{array}{l}\text { South }(3.0 \\
\mathrm{km})\end{array}$ & $0.892 \pm 0.073^{\mathrm{a}}$ & $* 0.980 \pm 0.014$ & $1.23 \pm 0.045$ & $0.561 \pm 0.026^{\mathrm{b}}$ & $0.713 \pm 0.026^{\mathrm{c}}$ & $0.860 \pm 0.062^{\mathrm{d}}$ & $0.710 \pm 0.055^{\mathrm{ab}}$ & $* 0.817 \pm 0.029^{c}$ & $0.961 \pm 0.031^{\mathrm{d}}$ \\
\hline $\begin{array}{ll}\text { East } & (0.5 \\
\mathrm{km}) & \end{array}$ & $0.943 \pm 0.070^{\mathrm{a}}$ & $* 1.104 \pm 0.043$ & $1.261 \pm 0.052^{\mathrm{b}}$ & $0.292 \pm 0.035$ & $0.555 \pm 0.041$ & $* 0.753 \pm 0.101$ & $0.780 \pm 0.043^{\mathrm{a}}$ & $* 0.892 \pm 0.062$ & $* 1.051 \pm 0.086^{\mathrm{b}}$ \\
\hline $\begin{array}{ll}\text { East } & (1.0 \\
\mathrm{km}) & \end{array}$ & $1.084 \pm 0.101^{\mathrm{a}}$ & $1.286 \pm 0.028$ & $1.655 \pm 0.072$ & $0.514 \pm 0.075$ & $0.762 \pm 0.030$ & $* 0.907 \pm 0.015$ & $0.936 \pm 0.071^{\mathrm{a}}$ & $1.035 \pm 0.091$ & $* 1.234 \pm 0.119$ \\
\hline $\begin{array}{ll}\text { East } & (3.0 \\
\mathrm{km}) & \end{array}$ & $0.684 \pm 0.029^{\mathrm{a}}$ & $* 0.812 \pm 0.040$ & $* 0.986 \pm 0.115$ & $0.232 \pm 0.013$ & $0.313 \pm 0.017$ & $0.410 \pm 0.032^{\mathrm{b}}$ & $0.507 \pm 0.025^{\mathrm{a}}$ & $* 0.589 \pm 0.045$ & $* 0.685 \pm 0.087^{\mathrm{b}}$ \\
\hline $\begin{array}{l}\text { West }(0.5 \\
\mathrm{km})\end{array}$ & $1.232 \pm 0.057$ & $1.540 \pm 0.046$ & $1.863 \pm 0.041$ & $* 0.808 \pm 0.017$ & $0.987 \pm 0.056^{\mathrm{a}}$ & $* 1.187 \pm 0.032$ & $* 1.009 \pm 0.075$ & $1.111 \pm 0.042^{\mathrm{a}}$ & $* 1.431 \pm 0.014$ \\
\hline $\begin{array}{l}\text { West } \quad(1.0 \\
\mathrm{km})\end{array}$ & $1.081 \pm 0.013$ & $1.261 \pm 0.046$ & $1.410 \pm 0.075$ & $0.709 \pm 0.059^{\mathrm{a}}$ & $* 0.754 \pm 0.042$ & $* 0.858 \pm 0.045$ & $0.761 \pm 0.039^{\mathrm{a}}$ & $* 0.914 \pm 0.041$ & $* 1.085 \pm 0.031$ \\
\hline $\begin{array}{l}\text { West }(3.0 \\
\mathrm{km})\end{array}$ & $* 0.756 \pm 0.042$ & $* 0.860 \pm 0.047$ & $* 0.983 \pm 0.116$ & $0.511 \pm 0.045^{\mathrm{a}}$ & $0.556 \pm 0.014^{\mathrm{b}}$ & $0.631 \pm 0.057^{c}$ & $* 0.542 \pm 0.034^{\mathrm{a}}$ & $* 0.657 \pm 0.072^{\mathrm{b}}$ & $* 0.730 \pm 0.071^{\mathrm{c}}$ \\
\hline
\end{tabular}

Table 3c. Seasonal variations in Copper $\left(\mathrm{mg} \mathrm{g}^{-1} \mathrm{DW}\right)$ in moss Hypnum cupressiforme Hedw from different transplant sites in Jammu during 2014, $2015 \& 2016$

\begin{tabular}{|c|c|c|c|c|c|c|c|c|c|}
\hline \multirow{2}{*}{$\begin{array}{c}\text { Samplin } \\
\mathbf{g} \\
\text { Sites } \\
\end{array}$} & \multicolumn{3}{|c|}{ Summer } & \multicolumn{3}{|c|}{ Monsoon } & \multicolumn{3}{|c|}{ Winter } \\
\hline & 2014 & 2015 & 2016 & 2014 & 2015 & 2016 & 2014 & 2015 & 2016 \\
\hline Baseline & $0.093 \pm 0.001$ & $0.119 \pm 0.005$ & $0.136 \pm 0.019$ & $0.008 \pm 0.001^{\mathrm{a}}$ & $0.012 \pm 0.005^{\mathrm{b}}$ & $0.026 \pm 0.011^{\mathrm{c}}$ & $0.014 \pm 0.005^{\mathrm{a}}$ & $0.022 \pm 0.006^{\mathrm{b}}$ & $0.035 \pm 0.018^{c}$ \\
\hline $\begin{array}{l}\text { North } \\
(0.5 \mathrm{~km})\end{array}$ & $0.636 \pm 0.056$ & $0.734 \pm 0.014$ & $\begin{array}{c}* 0.790 \pm 0.02 \\
7 \\
\end{array}$ & $* 0.309 \pm 0.016$ & $0.363 \pm 0.032$ & $\begin{array}{c}* 0.417 \pm 0.01 \\
8 \\
\end{array}$ & $* 0.433 \pm 0.016$ & $0.518 \pm 0.026$ & $* 0.593 \pm 0.031$ \\
\hline $\begin{array}{l}\text { North } \\
(1.0 \mathrm{~km})\end{array}$ & $0.735 \pm 0.054$ & $0.788 \pm 0.036$ & $\begin{array}{c}* 0.881 \pm 0.05 \\
6\end{array}$ & $* 0.408 \pm 0.029$ & $0.435 \pm 0.028$ & $0.477 \pm 0.050$ & $* 0.530 \pm 0.015$ & $0.582 \pm 0.043$ & $* 0.660 \pm 0.073$ \\
\hline $\begin{array}{l}\text { North } \\
(3.0 \mathrm{~km})\end{array}$ & $\begin{array}{c}* 0.309 \pm 0.036 \\
\text { a }\end{array}$ & $0.734 \pm 0.056$ & $\begin{array}{c}* 0.806 \pm 0.04 \\
2\end{array}$ & $\begin{array}{c}0.407 \pm 0.026^{\mathrm{a}} \\
\text { b }\end{array}$ & $0.435 \pm 0.059^{\mathrm{c}}$ & $0.580 \pm 0.028^{d}$ & $\begin{array}{c}* 0.464 \pm 0.015 \\
\text { b }\end{array}$ & $0.505 \pm 0.029^{\mathrm{c}}$ & $\begin{array}{c}* 0.630 \pm 0.071 \\
\mathrm{~d}\end{array}$ \\
\hline $\begin{array}{l}\text { South } \\
(0.5 \mathrm{~km})\end{array}$ & $0.093 \pm 0.019$ & $\begin{array}{c}* 0.587 \pm 0.03 \\
2\end{array}$ & $0.656 \pm 0.071^{\mathrm{a}}$ & $0.016 \pm 0.005^{\mathrm{b}}$ & $\begin{array}{c}* 0.337 \pm 0.033 \\
\mathrm{c}\end{array}$ & $\begin{array}{c}* 0.406 \pm 0.01 \\
4\end{array}$ & $0.034 \pm 0.002^{\mathrm{b}}$ & $0.471 \pm 0.049^{\mathrm{c}}$ & $* 0.582 \pm 0.029^{\mathrm{a}}$ \\
\hline $\begin{array}{l}\text { South } \\
(1.0 \mathrm{~km})\end{array}$ & $* 0.052 \pm 0.001$ & $1.232 \pm 0.116$ & $1.489 \pm 0.060$ & $0.011 \pm 0.001^{\mathrm{a}}$ & $* 0.707 \pm 0.042$ & $0.829 \pm 0.043$ & $* 0.023 \pm 0.001^{\mathrm{a}}$ & $* 0.932 \pm 0.056$ & $1.092 \pm 0.029$ \\
\hline $\begin{array}{l}\text { South } \\
(3.0 \mathrm{~km})\end{array}$ & $0.093 \pm 0.005$ & $\begin{array}{c}* 0.861 \pm 0.04 \\
7\end{array}$ & $1.041 \pm 0.028$ & $0.016 \pm 0.000$ & $0.584 \pm 0.027^{\mathrm{a}}$ & $0.743 \pm 0.028^{b}$ & $0.038 \pm 0.001$ & $* 0.661 \pm 0.053^{\mathrm{a}}$ & $0.815 \pm 0.031^{\mathrm{b}}$ \\
\hline $\begin{array}{l}\text { East }(0.5 \\
\mathrm{km})\end{array}$ & $0.755 \pm 0.028^{\mathrm{a}}$ & $0.831 \pm 0.056^{\mathrm{b}}$ & $0.987 \pm 0.072^{c}$ & $0.231 \pm 0.014$ & $0.363 \pm 0.017$ & $0.556 \pm 0.058$ & $0.636 \pm 0.057^{\mathrm{a}}$ & $0.760 \pm 0.028^{b}$ & $0.839 \pm 0.036^{\mathrm{c}}$ \\
\hline $\begin{array}{l}\text { East }(1.0 \\
\mathrm{km})\end{array}$ & $0.902 \pm 0.056^{\mathrm{a}}$ & $\begin{array}{c}1.028 \pm 0.01 \\
5 \\
\end{array}$ & $\begin{array}{c}* 1.230 \pm 0.07 \\
2 \\
\end{array}$ & $0.381 \pm 0.043$ & $0.504 \pm 0.028$ & $0.660 \pm 0.043$ & $0.784 \pm 0.043^{\mathrm{a}}$ & $* 0.904 \pm 0.044$ & $* 1.006 \pm 0.054$ \\
\hline $\begin{array}{l}\text { East }(3.0 \\
\mathrm{km})\end{array}$ & $* 0.581 \pm 0.044$ & $0.685 \pm 0.044$ & $\begin{array}{c}* 0.762 \pm 0.02 \\
8 \\
\end{array}$ & $0.182 \pm 0.013$ & $0.263 \pm 0.027$ & $0.310 \pm 0.029$ & $* 0.436 \pm 0.015$ & $0.471 \pm 0.043$ & $* 0.569 \pm 0.054$ \\
\hline $\begin{array}{l}\text { West }(0.5 \\
\mathrm{km})\end{array}$ & $0.957 \pm 0.059^{\mathrm{a}}$ & $1.335 \pm 0.019$ & $1.535 \pm 0.076$ & $0.689 \pm 0.016^{\mathrm{b}}$ & $0.861 \pm 0.063^{\mathrm{c}}$ & $0.982 \pm 0.054^{\mathrm{d}}$ & $0.837 \pm 0.045^{\mathrm{ab}}$ & $1.003 \pm 0.028^{\mathrm{c}}$ & $1.162 \pm 0.077^{\mathrm{d}}$ \\
\hline $\begin{array}{l}\text { West }(1.0 \\
\mathrm{km})\end{array}$ & $* 0.880 \pm 0.059$ & $1.056 \pm 0.026$ & $1.137 \pm 0.062$ & $0.610 \pm 0.044^{\mathrm{a}}$ & $0.661 \pm 0.075^{b}$ & $0.739 \pm 0.057^{\mathrm{c}}$ & $* 0.678 \pm 0.042^{\mathrm{a}}$ & $0.736 \pm 0.057^{b}$ & $0.857 \pm 0.045^{\mathrm{c}}$ \\
\hline $\begin{array}{l}\text { West }(3.0 \\
\mathrm{km})\end{array}$ & $* 0.657 \pm 0.056$ & $\begin{array}{c}* 0.735 \pm 0.05 \\
9\end{array}$ & $\begin{array}{c}* 0.836 \pm 0.03 \\
2\end{array}$ & $0.433 \pm 0.015^{\mathrm{a}}$ & $0.486 \pm 0.015^{\mathrm{b}}$ & $0.561 \pm 0.033^{\mathrm{c}}$ & $* 0.484 \pm 0.060^{\mathrm{a}}$ & $\begin{array}{c}* 0.535 \pm 0.030 \\
\text { b }\end{array}$ & $* 0.637 \pm 0.062^{c}$ \\
\hline
\end{tabular}


Table 3d. Seasonal variations in Cadmium ( $\mathrm{mg} \mathrm{g}^{-1} \mathrm{DW}$ ) in moss Hypnum cupressiforme Hedw from the different transplant of Jammu city during 2014, 2015 \& 2016

\begin{tabular}{|c|c|c|c|c|c|c|c|c|c|}
\hline \multirow{2}{*}{$\begin{array}{l}\text { Sampling } \\
\text { Sites }\end{array}$} & \multicolumn{3}{|c|}{ Summer } & \multicolumn{3}{|c|}{ Monsoon } & \multicolumn{3}{|c|}{ Winter } \\
\hline & 2014 & 2015 & 2016 & 2014 & 2015 & 2016 & 2014 & 2015 & 2016 \\
\hline Baseline & $0.007 \pm 0.000^{\mathrm{a}}$ & $0.013 \pm 0.004$ & $0.028 \pm 0.005$ & ND & ND & ND & ND & ND & ND \\
\hline $\begin{array}{l}\text { North }(0.5 \\
\mathrm{km})\end{array}$ & $0.111 \pm 0.014$ & $0.139 \pm 0.013$ & $0.184 \pm 0.029$ & $0.037 \pm 0.003^{\mathrm{a}}$ & $0.057 \pm 0.014^{b}$ & $0.061 \pm 0.015^{\mathrm{c}}$ & $0.059 \pm 0.011^{\mathrm{a}}$ & $0.060 \pm 0.017^{\mathrm{b}}$ & $0.080 \pm 0.013^{c}$ \\
\hline $\begin{array}{l}\text { North }(1.0 \\
\mathrm{km})\end{array}$ & $0.131 \pm 0.014$ & $0.140 \pm 0.012$ & $0.210 \pm 0.011$ & $0.055 \pm 0.002^{\mathrm{a}}$ & $0.063 \pm 0.002^{\mathrm{b}}$ & $0.082 \pm 0.002^{\mathrm{c}}$ & $0.060 \pm 0.006^{\mathrm{a}}$ & $0.079 \pm 0.001^{\mathrm{b}}$ & $0.134 \pm 0.013^{c}$ \\
\hline $\begin{array}{l}\text { North }(3.0 \\
\text { km) }\end{array}$ & $0.112 \pm 0.015$ & $0.134 \pm 0.014$ & $0.167 \pm 0.019$ & $0.034 \pm 0.006^{\mathrm{a}}$ & $0.055 \pm 0.014^{\mathrm{b}}$ & $0.061 \pm 0.006$ & $0.055 \pm 0.014^{\mathrm{a}}$ & $0.061 \pm 0.015^{\mathrm{b}}$ & $0.085 \pm 0.016$ \\
\hline $\begin{array}{l}\text { South }(0.5 \\
\mathrm{km})\end{array}$ & $* 0.053 \pm 0.031^{\mathrm{a}}$ & $0.106 \pm 0.014$ & $0.117 \pm 0.011$ & $* 0.022 \pm 0.005^{\mathrm{b}}$ & $0.043 \pm 0.006^{\mathrm{c}}$ & $0.066 \pm 0.003^{d}$ & $0.045 \pm 0.004^{\mathrm{ab}}$ & $0.058 \pm 0.002^{c}$ & $0.069 \pm 0.004^{\mathrm{d}}$ \\
\hline $\begin{array}{l}\text { South }(1.0 \\
\mathrm{km})\end{array}$ & $0.032 \pm 0.072^{\mathrm{a}}$ & $0.088 \pm 0.017$ & $0.109 \pm 0.001$ & $* 0.011 \pm 0.007$ & $0.027 \pm 0.002^{\mathrm{b}}$ & $0.036 \pm 0.002^{\mathrm{c}}$ & $* 0.028 \pm 0.006^{\mathrm{a}}$ & $0.038 \pm 0.004^{\mathrm{b}}$ & $0.046 \pm 0.003^{c}$ \\
\hline $\begin{array}{l}\text { South }(3.0 \\
\mathrm{km})\end{array}$ & $0.048 \pm 0.067^{\mathrm{a}}$ & $0.114 \pm 0.001$ & $0.137 \pm 0.002$ & $0.027 \pm 0.009$ & $0.035 \pm 0.003$ & $0.045 \pm 0.002$ & $0.034 \pm 0.016^{\mathrm{a}}$ & $0.058 \pm 0.003$ & $0.067 \pm 0.004$ \\
\hline $\begin{array}{ll}\text { East } & (0.5 \\
\mathrm{km}) & \\
\end{array}$ & $0.107 \pm 0.014$ & $0.136 \pm 0.011$ & $0.185 \pm 0.014$ & $0.021 \pm 0.001$ & $0.024 \pm 0.000$ & $0.034 \pm 0.002$ & $0.034 \pm 0.003$ & $0.054 \pm 0.014$ & $0.085 \pm 0.013$ \\
\hline $\begin{array}{ll}\text { East } & (1.0 \\
\mathrm{km}) & \end{array}$ & $0.115 \pm 0.013$ & $0.136 \pm 0.015$ & $0.160 \pm 0.019$ & $0.028 \pm 0.001^{\mathrm{a}}$ & $0.038 \pm 0.004^{\mathrm{b}}$ & $0.042 \pm 0.005^{\mathrm{c}}$ & $0.043 \pm 0.004^{\mathrm{a}}$ & $0.059 \pm 0.016^{\mathrm{b}}$ & $0.060 \pm 0.015^{\mathrm{c}}$ \\
\hline $\begin{array}{ll}\text { East } & (3.0 \\
\mathrm{km}) & \\
\end{array}$ & $0.089 \pm 0.016$ & $0.106 \pm 0.014$ & $* 0.117 \pm 0.018$ & $0.022 \pm 0.000^{\mathrm{a}}$ & $0.032 \pm 0.003^{b}$ & $0.041 \pm 0.004^{c}$ & $0.027 \pm 0.001^{\mathrm{aNS}}$ & $0.037 \pm 0.006^{\mathrm{b}}$ & $* 0.059 \pm 0.014^{\mathrm{c}}$ \\
\hline $\begin{array}{l}\text { West } \quad(0.5 \\
\mathrm{km})\end{array}$ & $0.063 \pm 0.015$ & $0.089 \pm 0.016^{\mathrm{a}}$ & $0.129 \pm 0.015$ & $0.016 \pm 0.001^{\mathrm{b}}$ & $0.035 \pm 0.004^{c}$ & $0.040 \pm 0.005^{\mathrm{d}}$ & $0.026 \pm 0.001^{\mathrm{bNS}}$ & $0.056 \pm 0.001^{\mathrm{ac}}$ & $0.061 \pm 0.014^{\mathrm{d}}$ \\
\hline $\begin{array}{l}\text { West }(1.0 \\
\mathrm{km})\end{array}$ & $0.084 \pm 0.014$ & $0.111 \pm 0.001$ & $0.137 \pm 0.017$ & $0.035 \pm 0.005^{\mathrm{a}}$ & $0.038 \pm 0.003^{\mathrm{b}}$ & $0.055 \pm 0.014^{\mathrm{c}}$ & $0.037 \pm 0.004^{\mathrm{a}}$ & $0.058 \pm 0.014^{\mathrm{b}}$ & $0.059 \pm 0.014^{c}$ \\
\hline $\begin{array}{l}\text { West }(3.0 \\
\mathrm{km})\end{array}$ & $0.107 \pm 0.017$ & $0.113 \pm 0.016^{\mathrm{a}}$ & $0.155 \pm 0.014$ & $0.039 \pm 0.001^{\mathrm{b}}$ & $0.054 \pm 0.002^{\mathrm{c}}$ & $0.063 \pm 0.002^{\mathrm{d}}$ & $0.058 \pm 0.014^{\mathrm{b}}$ & $0.081 \pm 0.012^{\mathrm{ac}}$ & $0.086 \pm 0.014^{d}$ \\
\hline
\end{tabular}

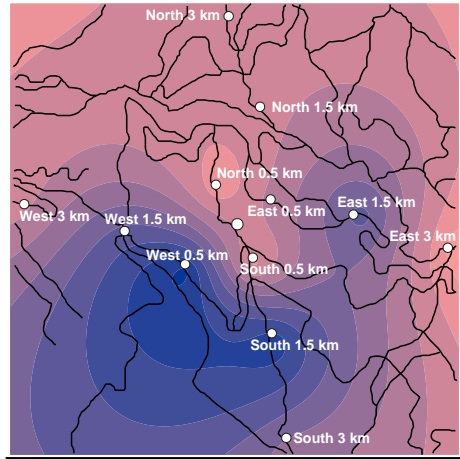

Fig. 2a Annual average Lead $\left(\mathrm{mg} \mathrm{g}^{-1}\right.$ DW) during summer 2014-2016 at Jammu in Hypnum cupressiforme Hedw

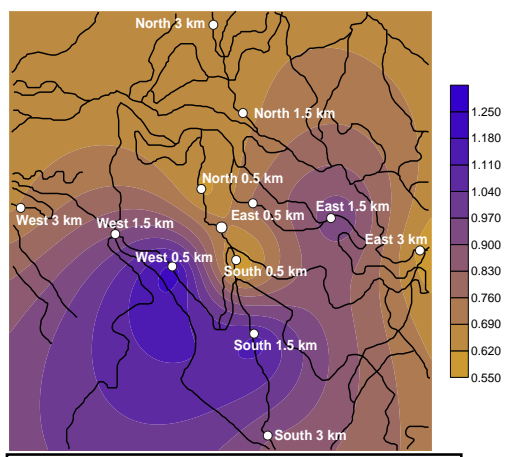

Fig. 3a Annual average copper $\left(\mathrm{mg} \mathrm{g}^{-1}\right.$ DW) during summer 2014-2016 at Jammu in Hypnum cupressiforme Hedw

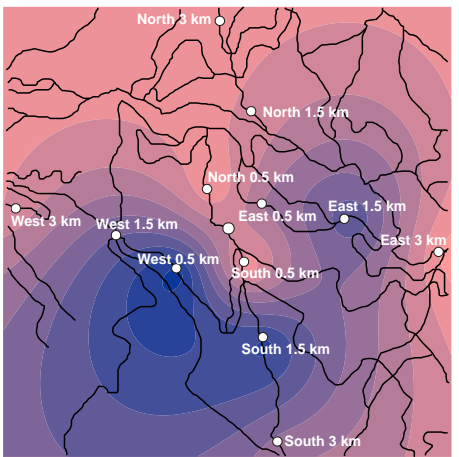

Fig. 2b Annual average Lead ( $\mathrm{mg} \mathrm{g}^{-1}$ DW) during monsoon 2014-2016 at Jammu in Hypnum cupressiforme Hedw

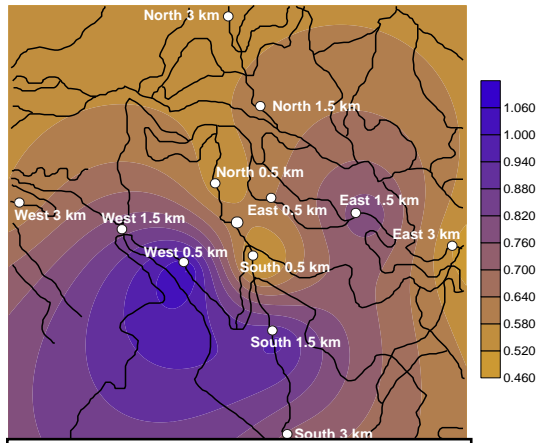

Fig. 3b Annual average copper $\left(\mathrm{mg} \mathrm{g}^{-1}\right.$ DW) during monsoon 2014-2016 at Jammu in Hypnum cupressiforme Hedw

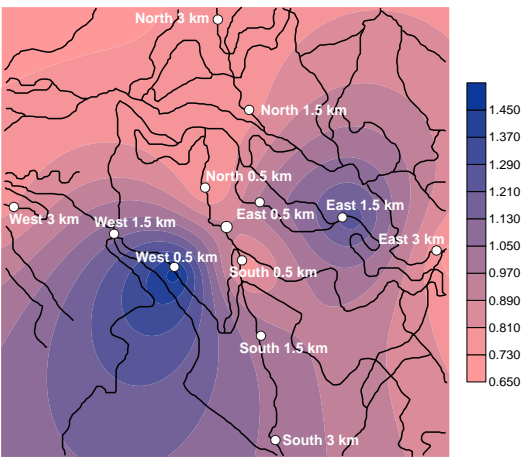

Fig. 2c Annual average Lead ( $\mathrm{mg} \mathrm{g}^{-1}$ DW) during winter 2014-2016 at Jammu in Hypnum cupressiforme Hedw

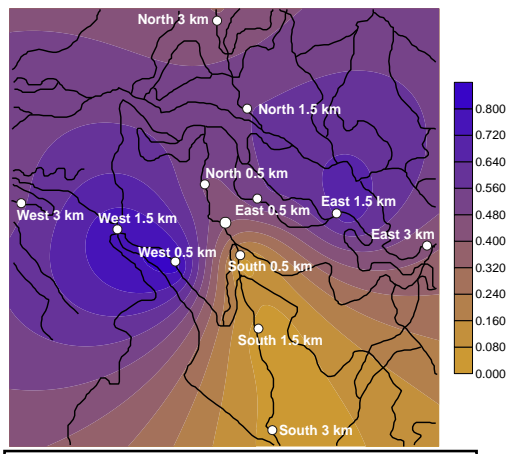

Fig. 3c Annual average copper $\left(\mathrm{mg} \mathrm{g}^{-1}\right.$ DW) during winter 2014-2016 at Jammu in Hypnum cupressiforme Hedw
$\mathrm{Cu}$ concentrations show significantly different values over baseline concentration at all sampling sites in all three consecutive years (Table $3 \mathrm{c}$ ). Statistically significant variation
( $\mathrm{p} \leq 0.05$ ) was detected at all distances towards the north direction from baseline in the year 2016 during summer, monsoon, and winter sampling seasons. The average annual 
concentration was measured as $0.828 \mathrm{mg}$ g-1 DW in 2014 and $1.066 \mathrm{mg} \mathrm{g}-1 \mathrm{DW}$ in 2005 towards the west at $0.5 \mathrm{~km}$, whereas $1.136 \mathrm{mg}$ g-1 DW in 2016 at south $1 \mathrm{~km}$. The low concentration was measured in moss samples harvested from different sites of the study areas i.e., at south at the distance of $1 \mathrm{~km}(0.028 \mathrm{mg} \mathrm{g}-1 \mathrm{DW})$ in 2014 , at the south side at the distance of $0.5 \mathrm{~km}(0.535 \mathrm{mg}$ g- $1 \mathrm{DW})$ in 2015 and in east nearly at $3 \mathrm{~km}(0.649 \mathrm{mg} \mathrm{g}-1 \mathrm{DW})$ in 2016. (Fig. 3a-3c). The values of $\mathrm{Zn}, \mathrm{Pb}$ and $\mathrm{Cu}$ analyzed from moss transplants samples collected from the control site of Manesar Lake, do not show a significant difference $(p \leq 0.01)$ between the monsoon and winter seasons in all three consecutive years. However, at the same time concentration of $\mathrm{Cd}$ was below the detection limit (ND) for the monsoon and winter season in 2014, 2015 even in 2016, including non-significant values between summer of 2014 and monsoon of 2016 along with the monsoon and winter of 2016 (Table 3d).

Analytical results of $\mathrm{Cd}$ of moss bag of Hypnum cupressiforme exhibited non-significant values ( $\mathrm{p} \leq 0.01,0.05)$ at some places during winter 2014 in comparison to baseline concentration (Table 3d). A significant difference for $\mathrm{Cd}$ at $\mathrm{p} \leq 0.05$ levels was shared by summer and monsoon towards south at $0.5 \mathrm{~km}$ while during monsoon and winter $\mathrm{Cd}$ data exhibited same pattern at south but at $1 \mathrm{~km}$. The annual average distribution pattern was high towards north at $1 \mathrm{~km}$ from baseline $(0.081$ mg g- ${ }^{1} \mathrm{DW}, 0.094 \mathrm{mg} \mathrm{g-}^{1} \mathrm{DW}$ and $\left.0.142 \mathrm{mg} \mathrm{g-}^{1} \mathrm{DW}\right)$ and was low at $1 \mathrm{~km}$ towards south $\left(0.023 \mathrm{mg} \mathrm{g-}^{1} \mathrm{DW}, 0.051 \mathrm{mg} \mathrm{g-}{ }^{1}\right.$ DW and $0.063 \mathrm{mg} \mathrm{g-}{ }^{1}$ DW) during 2014, 2015 and 2016 respectively

\section{Seasonal percentage increase in zinc}

It has been observed that the increase in the concentration of zinc was significantly high during the monsoon season in all the directions except West of the baseline (Fig. 4).

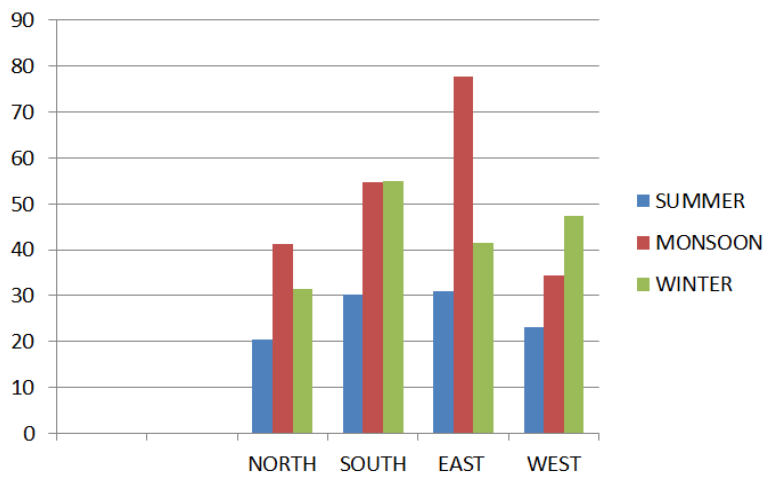

Fig. 4 Percentage increase in concentration of Zinc in moss Hypnum cupressiforme Hedw from different transplant sites in Jammu during year 2014, 2015 \& 2016 in different direction

\section{Seasonal percentage increase in lead}

It has been observed that the increase in the concentration of lead was prominent during the monsoon season in all the directions except $3 \mathrm{~km}$ West of the baseline (Fig. 5).

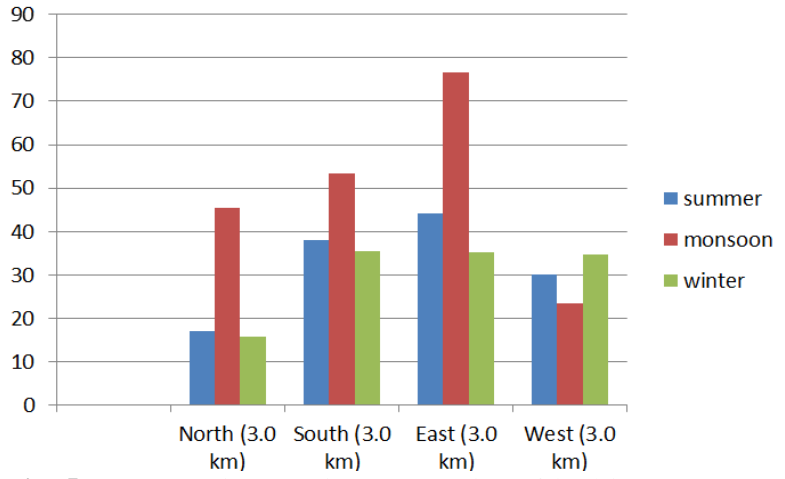

Fig. 5 Percentage increase in concentration of lead in moss Hypnum cupressiforme Hedw from different transplant sites in Jammu during the years $2014,2015 \& 2016$ in different direction

\section{Seasonal percentage increase in copper}

The increase in the concentration of copper was abnormally high during the monsoon season along $3 \mathrm{~km}$ south of the baseline (Fig. 6).

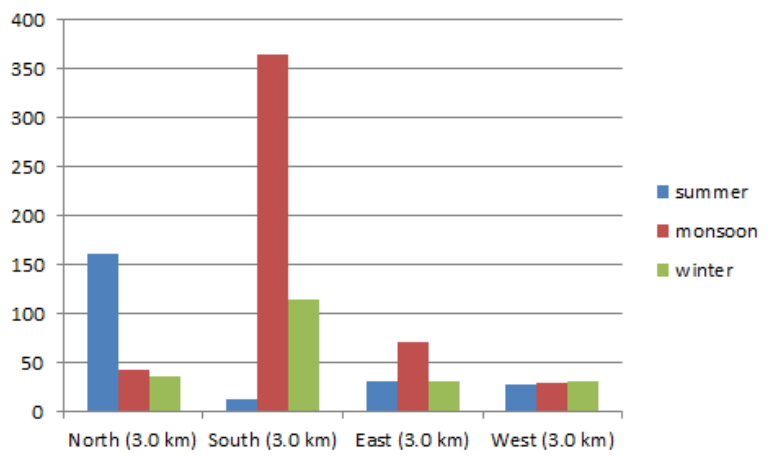

Fig. 6 Percentage increase in concentration of copper in moss Hypnum cupressiforme Hedw from different transplant sites in Jammu during year $2014,2015 \& 2016$ in different direction

\section{Seasonal percentage increase in cadmium}

It is observed that the percentage increase in concentration of cadmium during the summer season is maximum along $3 \mathrm{~km}$ South whereas during monsoon and winter season it is maximum in east.

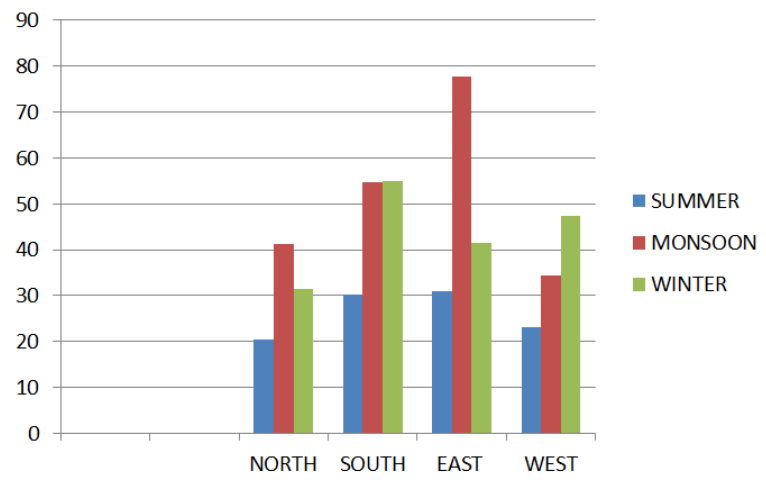

Fig. 7 Percentage increase in concentration of cadmium in moss Hypnum cupressiforme Hedw from different transplant sites in Jammu during year 2014, 2015 \& 2016 in different direction 


\section{Seasonal variation of metals along $3 \mathrm{~km}$ North}

During summer and winter seasons gradual increase in the concentration of metals $(\mathrm{Zn}, \mathrm{Pb}, \mathrm{Cu}$, and $\mathrm{Cd})$ was reported during 2014-2016 except monsoon season (Fig. 8a). The concentration of copper was reported to be high along $3 \mathrm{~km}$ North of baseline during the monsoon season.

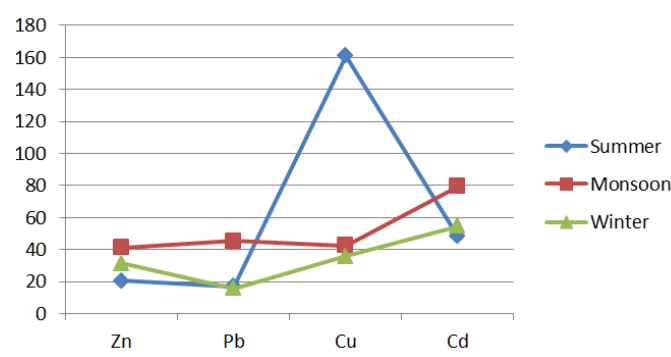

Fig. 8a increase in conc. of metals during $3 \mathrm{~km}$ North

\section{Seasonal variation of metals along $3 \mathrm{~km}$ South}

During summer season cadmium was increased to the maximum in moss Hypnum cupressiforme Hedw (Fig. 8b). The concentration of copper was reported to be abnormally high along $3 \mathrm{~km}$ South of the baseline during the monsoon season. On the other hand, cadmium shows the least increase during the monsoon season.

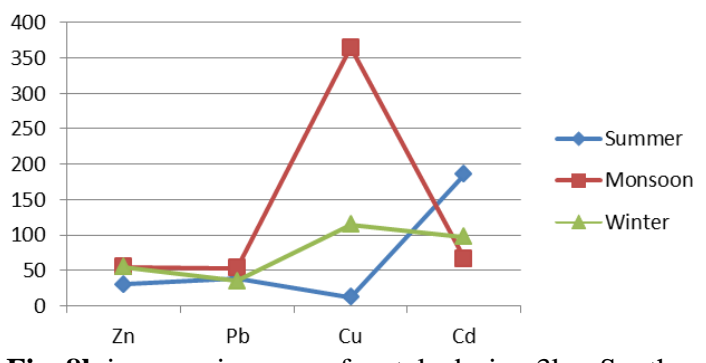

Fig. $8 \mathbf{b}$ increase in conc. of metals during $3 \mathrm{~km}$ South

\section{Seasonal variation of metals along $3 \mathrm{~km}$ East}

The percentage increase in zinc, lead and copper was studied and analyzed. It was measured to be appreciably high during the monsoon season for the year 2014-2016 along $3 \mathrm{~km}$ east (Fig. 8c). This could be due to the precipitation, as metals do not get dispersed in the air during the monsoon season and are taken up by plants as wet deposition along with droplets. Only cadmium was found to show higher levels during winter season. Metal concentration was high in transplants harvested in proximity to the baseline area over exposed moss transplants harvested at the distance of $3 \mathrm{kms}$.

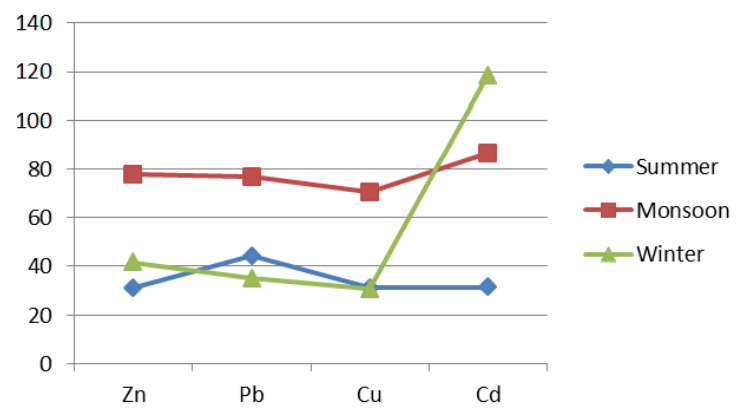

Fig. 8c increase in conc. of metals during $3 \mathrm{~km}$ East

\section{Seasonal variation of metals along $3 \mathrm{~km}$ West}

Similar trends were observed for different seasons. For the increase in concentration of metals $(\mathrm{Zn}, \mathrm{Pb}, \mathrm{Cu}, \mathrm{Cd})$ in moss Hypnum cupressiforme Hedw (Fig. 8d), when measured along $3 \mathrm{~km}$ west of the baseline during 2014-2016, high concentration of cadmium during monsoon season (Fig. 8d) could not be ruled out due to e-waste generated due to excessive usage of electronic gadgets.

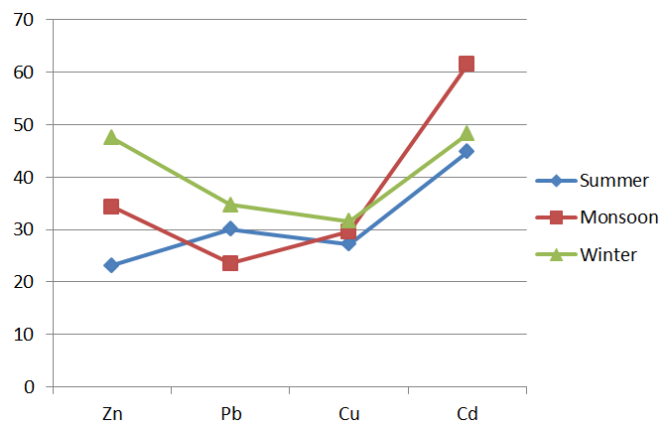

Fig. 8d increase in conc. of metals during $3 \mathrm{~km}$ west of Baseline

\section{Discussion}

Our results clearly showed that moss Hypnum cupressiforme Hedw increased in element content after exposure in the under taken areas of Jammu district than base line of forest cover treated as control.

The increase of element content in exposed moss is related to both (i) bioaccumulation ability of moss and (ii) and local heavy metal pollution and its source. Study also confirm the effectiveness of bioaccumulation of moss Hypnum cupressiforme Hedw which is also documented earlier [37, 38].

It is also concluded that moss analysed from control or forest cover sites was not affected by elemental contamination, on the contrary exposed moss Hypnum cupressiforme Hedw from vicinity of monitoring sites had high elemental load. Same could be due to monitoring locations were much crowded and had more traffic congestion, suggest moss is reliable and inexpensive tool for evaluation of the environment

Gradient of the metal load from different transplant sites covering all four directions of Jammu during the year 2016 over 2014 and 2015 were in order of $\mathrm{Cu}>\mathrm{Cd}>\mathrm{Zn}>\mathrm{Pb}$.

It is also demonstrated that percent increases for metals under taken after exposure of 4 months corresponding to summer and again exposure for 4 months corresponding to the winters, and subsequent exposure of 4 months represent monsoon were in order of $\mathrm{Zn}, \mathrm{Pb}, \mathrm{Cu}$, and $\mathrm{Cd}$.

An increase in mean lead concentration during monsoon in proximity to the city may be attributed to many-fold increase in tourist movement results into very high vehicular traffic. (Table 2). During the monsoon season, low or nil wind current does not allow the pollutants go away consequently lead is not dispersed in the air and is precipitated in the soil which is eventually taken up by the plants.

Nevertheless vehicles are using unleaded fuels, $\mathrm{Pb}$ is still the main polluting element measured in urban environment, 
could not be ruled out originating from other source too i.e. fuel combustion, wear and tear of automobile parts, electronic batteries, abrasion of tyres, automobile parts, brakes and engines.

An increase in metal load during summer towards North (Fig. 8a) could be due to high temperature along with low rainfall influence dry deposition and decrease hydration.

The analysis of transplanted moss bags exhibited significant seasonal variation in metal concentration in the moss Hypnum cupressiforme Hedw, which could be due to local sources [37, 38]. Significantly higher concentration of metals $(\mathrm{Zn}, \mathrm{Pb}, \mathrm{Cu}$ and $\mathrm{Cd}$ ) in moss at Jammu city during summer season at different catchments sites except at Manesar lake site could be due to high volume of tourist inflow as Jammu is a religious place. There is a high inflow of devotees and pilgrims, leading to heavy traffic which, subsequently results in high emissions of pollutants [39]. Airborne road dust plays a key role in pollution and its uptake by plants [40]. Other sources can not be ruled out i.e., abrasion from automobile tyres resulted in many folds increase in atmospheric metal load [37]. Present findings are in agreement that these sources are automobile emissions [40, 41]. As this region is devoid of industries, contribution to the air pollution from industries is negligible [42]. Seasonal variations could be due to (i) low tourist inflow in winters and (ii) due to a drop in atmospheric temperature in the range of 2 to $10{ }^{\circ} \mathrm{C}$ resulting in condensation of water droplets which does not allow pollutant to mix with air [43]. Drop-in metal values in monsoon could be because the pollutants are washed off with rainwater. Present findings are in agreement with the report of Carlo et al [43], where they reported seasonal variations due to different reasons. In the present study, it is safe to say that seasonal variations do affect in elemental concentration of the moss in a country like India, where three different seasons are well documented. Municipal wastes, exhaust from automobiles, batteries, construction material, paints and pigments, etc. seem to be the major sources of $\mathrm{Zn}$ in the atmosphere.

Values of calculated metals were approximately the higher than level measure as in other neighboring states $[11,37]$. No long-range transmission of air pollutants (metals) observed.

Decrease in atmospheric metal concentration has been observed in entire Europe (Ruhling and Steinnesm 1998). On the contrary, atmospheric heavy metal concentrations in different parts of India have an increasing trend except during pandemic period constant [32 ].

The present study strongly validates the moss Hypnum cupressiforme Hedw for bio mapping studies of atmospheric metal, their dispersion and their possible sources. Studies are part of the monitoring program and results are in agreement with previous reports [32].

\section{Conclusion}

To achieve the temporal trend of atmospheric metal precipitation, widely distributed moss species Hурпит cupressiforme Hedw shortlisted and validated for metal tolerance. Moss bags were transplanted at equidistance in all four directions (north, south, east and west) during winters, summer and monsoon for measuring atmospheric metals from Jammu.

The overall gradient of the metal load was in the order of $\mathrm{Cu}>\mathrm{Cd}>\mathrm{Zn}>\mathrm{Pb}$ during the study period of three years. Nevertheless total average percentage increase for the metals $\mathrm{Zn}, \mathrm{Pb}, \mathrm{Cu}$, and $\mathrm{Cd}$, was $40 \%, 37 \%, 79 \%$, and $76 \%$. Distance dependent metal dispersion graph revealed strong metal load in proximity to the source. The high concentration of all metals were measured in moss transplants harvested from distance of $1 \mathrm{~km}$ over $3 \mathrm{~km}$ from baseline. Analyzed metal values in moss transplant during different season were not constant. The result obtained after analysis suggests the potential of moss Hypnum cupressiforme as reliable biomonitor of atmospheric deposition for elements and its suitability being efficient accumulator. This also implies that the active transplant technique is a cost effective and reliable tool for mapping of temporal trends

\section{Acknowledgments}

The author is thankful to the State Council of Science and Technology, Lucknow for their financial support. Thanks are also due to the Department of Biotechnology for providing funds for the fabrication of AAS. Help rendered by Bareilly College, Bareilly is gratefully acknowledged. The author acknowledges the help extended by Professor Yash Pal Sharma, head of the Botany Department, during a field trip to Jammu.

\section{References}

[1] Loppi S. Contardo T. Winkler A. Lichen biomonitoring and environmental justice a case study from Milan, 2021. 34 ICP meet, ITALY pp 11.

[2] Frontasyeva MV., Steinnes E. and Harmens H. Monitoring long-term and large-scale deposition of air pollutants based on moss analysis. Chapter in a book "Biomonitoring of Air Pollution Using Mosses and Lichens: Passive and Active Approach - State of the Art and Perspectives", Edts. M. Aničić Urošević, G. Vuković, M. Tomašević, Nova Science Publishers, New-York, USA, 2016, pp. 246.

[3] Fernandez J. A. Aboal J R. \& Caballeira A. Identification of pollution sources by means of moss bags. Ecotoxicology and. Environment Safety. 2004, 59 (1): pp 76-83. DOI: 10.1016/j.ecoenv.2004.01.007.

[4] Steinnes E. A critical evaluation of the use of naturally growing moss to monitor the deposition of atmospheric metals. The Science of the Total Environment. 1995, pp 243-249. https://doi.org/10.1016/0048-9697(95)04360-D

[5] Ho YB. Tai KM. Potential use of a roadside fern (Pteris vittata) to biomonitor $\mathrm{Pb}$ and other aerial metal deposition. Bulletin of Environmental Contamination and Toxicology 1985, 35: pp 430-438. https://doi.org/10.1007/BF01636534.

[6] Ho YB. \& Tai KM. Elevated levels of lead and other metals in roadside soil and grass and their use to monitor aerial metal depositions in Hong Kong, Environmental Pollution 1988. 49: pp 37-51. DOI: 10.1016/0269-7491(88)90012-7

[7] Kovács M. Editor. Biological indicators in environmental protection. Akadémiai Kiadó, Budapest. 1992.

[8] Markert B. Herpin U. Berlekamp J. Oehlmann J. Grodinska K. Mankovska. B. A comparison of heavy metal deposition in selected Eastern European countries using the moss monitoring method, with special emphasis on the Black Triangle. The Science of the Total Environment, 1996, 193: 85-100. https://doi.org/10.1016/S0048-9697(96)05333-8 
[9] Saxena D K and Harinder. Uses of Bryophytes. Resonance 2004. 9: pp56-65

www.ias.ac.in/article/fulltext/reso/009/06/0056-0065990.

[10] Glime JM and Saxena DK, Uses of Bryophytes, Today and Tomorrow Printers and Publishers, 1991. New Delhi, India.

[11]. Saxena, DK. Singh S. \& Srivastava K. Atmospheric Heavy Metal Deposition in Garhwal Hill Area (India): Estimation Based on Native Moss Analysis. Aerosol and Air Quality Research. 2008. 8 (1) pp 94-111. https://doi.org/10.4209/aaqr.2007.07.0031

[12] Saxena Dinesh K, Saiful A. metal deposition pattern in Kumaon hills (India) through active monitoring using moss Racomitrium Crispulum, Iran. J. Environ. Health. Sci. Eng 2010, Vol. 7, No. 2, pp. 103-114

[13] Catalin DB. Passive biomonitoring, of atmospheric pollution with heavy metals using native epigenetic moss. 2009. Conference: International Conference on Environmental Engineering and Management (ICEEM 05/2009) September 2009.

[14] Shotbolt L. Buker P. Ashmore MR. Reconstructing temporal trends in heavy metal deposition: assessing the value of herbarium moss samples. Environmental Pollution. 2007. 147(1) pp 120-130, DOI:10.1016/j.envpol.2006.08.031.

[15] Saxena, DK, S. Singh \& K. Srivastava. Moss bag technique for monitoring metal precipitation. Environment Conservation Journal. 2007. 8 (2) pp16-21.

[16[ Kosior G. Samecka-Cymerman Kolon, A, Kempers, AJ. Bioindication capacity of metal pollution of native and transplanted Pleurozium schreberi under various levels of pollution. Chemosphere. $2010 \quad 81, \quad 3$ pp321-326. DOI: 10.1016/j.chemosphere.2010.07.029

[17] Zulema V, Sofía D, Dinesh KS, J R. Aboal and Fernández. J. Á Levels of Antioxidant Compound Glutathione in Moss from Industrial Areas. Atmosphere 2018, 9(7), 284; doi.org/10.3390/atmos9070284 (registering DOI)

[18] Harmens H. Mosses as biomonitors of atmospheric heavy metal deposition: Spatial patterns and temporal trends in Europe. October 2010 Environmental Pollution Environmental Pollution 158(10):3144

3156.DOI:10.1016/j.envpol.2010.06.039

[19] Berg T. \& Steinnes. E. Use of mosses (Hylocomium splendens and Pleurozium schreberi) as biomonitors of heavy metal deposition: From relative to absolute deposition values Environmental Pollution. 1997, Volume 98, Issue 1, pp 61-71. https://doi.org/10.1016/S0269-7491(97)00103-6

[20] Schroder W. \& Pesch R... Integrative Monitoring Analysis Aiming at the Detection of Spatial and Temporal Trends of Metal Accumulation in Mosses. Journal of Atmospheric Chemistry 2004:43(1-3) pp 23-38. DOI:10.1007/s10874004-1212-0
[21] Sloof J. Bruin E. De and Wolterbeek HTh. Critical evaluation of some commonly used biological monitors for heavy metal pollution, Proc. Third International Conference Environment Contamination, 1998, .26-29 September, Venice, 296-298

[22] Ares A, JR. Aboal, A, Carballeira S, Giordano P. Adamo, \& JÁ Fernández. Moss bag biomonitoring: a methodological review. Science of the Total Environment 2012, 432, pp 143158

[23] Stirbet A. Lazar D. Kromdijk, J. and Govindjee G. 2018. Chlorophyll a fluorescence induction: can just a second measurement be used to quantify abiotic stress response. Photosynthetica 2018, 56(1):86-104 | DOI: 10.1007/s11099018-0770-3.

[24] Beliaeff B. and Burgeot. T. Integrated biomarker response: a useful tool for ecological risk assessment. Environmental Toxicology and Chemistry 2002, 21(6):13161322 , DOI:10.1897/1551-5028(2002)021<1316: IBRAUT>2.0.CO;2

[25] Gangulee HC. Mosses of Eastern India and Adjacent Regions. Kolkata, India: Estend Printers. 1969-1980. Vol. IIII (Fasc. 1-8.).

[26] Chopra R. S. Taxonomy of Indian mosses. Publication and information directorate, C S I R, New Delhi. 1975.

[27] Frahm JP. A preliminary study of the infraspecific taxa of Hypnum cupressiforme in Europe. Arch. Bryol. 2009; 40 pp $1-10$.

[28] Riaz M. Sharma A and Langer A. A preliminary study on the moss flora of Kishtwar, J\&K (North-West Himalaya). Journal of Plant Development Sciences 2015. 7: pp 737-742. Google Scholar

[29] Baker NR. Chlorophyll fluorescence: a probe of photosynthesis in Vivo. Annu. Rev. Plant. Biol. 2008, 59, pp89-113. DOI: 10.1146/annurev.arplant.59.032607.092759

[30] Schreiber, U., W. Bilger, \& C. Neubauer. 1995. Chlorophyll fluorescence as a non-intrusive indicator for rapid assessment of in vivo photosynthesis. Ecophysiology of Photosynthesis pp 49-. In: Schulze, E.D. \& M. M. Caldwell (eds.) Ecophysiology of photosynthesis. Berlin: SpringerVerlag

[31] Yang-Er Chen, Nan Wu, Zhong-wel Zhang, Ming Yuan abd Shu Yuan. 2019. Perspective of monitoring heavy metals by moss visible chlorophyll fluorescence parameters. Frontiers in Plant Sciences. January 2019, Vol 10 Article 35, https://DOI:10.3389/fpls.2019.00035

[32]. Saxena DK and Saxena A, Atmospheric metal load by mosses of pre and post pandemic periods. In 34th Task Force Meeting, ICP vegetation, 22-25 February 2021, pp 54

[33]. Saxena DK Sharma BK, Srivastava HS, Anuj Saxena, Niharika Shankar, Kamakshi, Adrash Goswami. Need of bryophyte bank for environmental monitoring in India, Current Science, 2001. 81, No 11pp 1395- 1396, 10 December. https://www.jstor.org/stable/24106553 
[34]. Karmer CY. Extension of multiple range tests to group means with unequal numbers of replication. Biometrics. 1956; 12: pp 307-310. https://doi.org/10.2307/3001469

[35]. Snedecor G W \& Cochran W G. Statistical methods. Lowa State University, U. S. A. Oxford and I. B. H. Publishing Co., 1967. New Delhi.

[36]. Amarpreet, K Anju Rao and Sunita Kapila. Taxonomic Study of some Mosses of Doodhpathri (Budgam) J\&K, India. International Journal of Advances in Pharmacy, Biology and Chemistry 2015 Vol. 4(1), Jan - Mar, 196-208. http://www.ijapbc.com/ .

[37] Saxena DK and Arfeen S. metal deposition pattern in Kumaon hills (India) through active monitoring using Racomitrium crispulum. Iran. J. Environ Health Sci. Eng, 2010, Vol 7, pp103-114.

[38] Saxena DK, Hazem MK. and Dheeraj G. Large scale atmosphere monitoring by mosses in India: A CONCEPTUAL APPROACH in proceeding of 24th Task Force Meeting of the ICP Vegetation 31-3 rd February 2011 pp 21 edited by Harmens H. Mills G \& Nina Menichino), Rapperswil-Jona, Switzerland,

http://nora.nerc.ac.uk/id/eprint/13976/1/Book_of_abstracts.pdf

[39] Rong Hu, Yun Yan, Xiaoli Zhou Yanming Fang. Monitoring Heavy Metal Contents with Sphagnum Junghuhnianum Moss Bags in relation to Traffic Volume in Wuxi, China. International Journal of Environmental Research

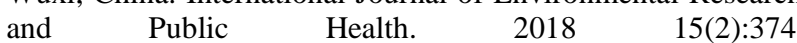
DOI:10.3390/ijerph15020374.

[40] Huang X. Olmez I. Aras NK. \& Gordan GE. Emissions of trace elements from motor vehicles: potential marker elements and source composition profile. The Atmospheric Environment 1994.28 (8): $\quad$ pp 1358-1391. www.sciencedirect.com/science/journal/13522310

[41] Santelmann MV \& Gorham. E. The influence of airborne road dust on the chemistry of Sphagnum mosses. Journal of Ecology, 1988, 76pp 1219-1231

[42] Zechmeister, H.G., A. Riss and A. Hanus-Illnar. 2014. Biomonitoring of atmospheric heavy metal deposition by mosses in the vicinity of industrial sites. Journal of Atmospheric Chemistry 43(1-3): 461-477

[43] Carlo Barbante, Claude Boutron, cd Christine Morel, c Christophe Ferrarice Jean Luc Jaffrezo, c Giulio Cozzi, a Vania Gasparia and Paolo Cesconab, Seasonal variations of heavy metals in central Greenland snow deposited from 1991 to 1995,2003 Issue

2003.https://pubs.rsc.org/en/content/articlelanding/2003/em/b2

10460a\#!divAbstract

[44]. Conard HS. Redfearn John F. Bamrick (Editor), Edward T. Cawley. How to know the mosses and liverworts. Revised Edition. Wm. C. Brown. 1979 pp 302 